\title{
Two Diffusion Kurtosis Imaging Post-processing Methods for Differentiating Glioma Grades, IDH Mutation Statuses, and Heterogeneity
}

Jie Bai

the first affiliated hospital of zhengzhou university

Ankang Gao

the first affiliated hospital of zhengzhou univercity

Yuan Hong

School of Mathematical Sciences of zhejiang univercity

GuoHua Zhao

the first affiliated hospital of zhengzhou university

Yong Zhang

the first affiliated hospital of zhengzhou university

Dexing Kong

school of mathematical sciences,zhejiang university

Jingliang Cheng ( $\nabla$ fccchengj|@zzu.edu.cn )

the first affiliated hospital of zhengzhou university

Research article

Keywords: diffusion magnetic resonance imaging, heterogeneity, glioma, isocitrate dehydrogenase

Posted Date: November 24th, 2020

DOl: https://doi.org/10.21203/rs.3.rs-112555/v1

License: (c) (i) This work is licensed under a Creative Commons Attribution 4.0 International License.

Read Full License 


\section{Abstract}

Background: To compare the application of two DKI post-processing methods that DKE software and DKI histogram analysis in glioma grading, IDH mutation typing, and evaluation of tumor heterogeneity.

Methods: Patients who underwent surgery and were pathologically diagnosed with glioma after MR DKI scan. DKE software was used to calculate diffusion parameters, including fractional anisotropy, mean kurtosis (MK), radial kurtosis, and axial kurtosis. Histogram parameters were calculated, including minimum, maximum, mean, standard deviation, percentile values (25th, 50th, 75th, 95th), kurtosis, and skewness of Kapp and Dapp. The ROls of the two post-processing methods were consistently and manually selected in continuous solid tumor regions. According to the result of Kolmogorov-Smirnov (KS) test, Independent-samples T test or Mann - Whitney - Wilcoxon test was used to distinguish glioma grads. The parameters with the best percentile were identified by analysis of the area under the curve (AUC) of the receiver operating characteristic (ROC) analysis.

Results: Seventy-three patients with glioma were observed, including 21 with low-grade gliomas (WHO II) and 52 with high-grade gliomas (WHO III, n=13; WHO IV, n=39), 38 of whom had IDH mutation status. There were significant differences between the high- and low-grade glioma groups regarding the maximum, mean, standard deviation, $\mathrm{C75}$, and $\mathrm{C} 95$ of the Kapp values and the minimum, mean, C25, C50, C75, C95, and skewness of the Dapp values. The MK values were significantly different among the WHO II, III, and IV grades. MK, mean Kapp, and C75 and C95 of the Kapp could be used to predict IDH mutations in patients with glioma.

Conclusions: Several quantitative DKI parameters obtained from the DKE software and histogram analysis could be used for glioma grading and predicting IDH mutations. However, DKI histogram analysis was useful for glioma heterogeneity.

\section{Background}

Cerebral gliomas are the most common malignant tumors of the CNS. With genotyping was included in glioma diagnosis, isocitrate dehydrogenase (IDH) as an important maker has been focused, but there was no change in glioma grading [1]. Patients with high-grade glioma (WHO $\mathbb{-}-\mathbb{Z}$ ) usually have a poorer

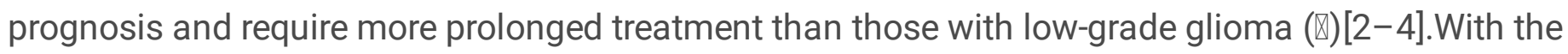
increase of tumor grade, the tumor heterogeneity increase, which include cellular proliferation, necrosis, differences in blood flow and angiogenesis, cellular metabolism, hypoxia and so on, and it has also been postulated that increased image heterogeneity [5-6].

As is known, MRI is the preferred imaging for glioma diagnosis. Among MRI sequences, the non-Gaussian diffusion model-based diffusion kurtosis imaging (DKI) [7], which been known as can reflect the difference of water diffusion of microstructures [8]. Diffusional Kurtosis Estimator (DKE)[9] is one of common DKI postprocess, which could got mean kurtosis (MK), axial kurtosis (AK) and radial kurtosis (RK) to reflect the diffusion kurtosis of the tissue[10]. Current research has shown that the MK value is the 
most valuable DKI parameter for glioma grading [11], and a marker for predicting survival and the IDH status in glioma research [12-13]. Histogram analysis which based on grey level frequency distribution could provide more information regarding quantitative parameters image, include percentile, mean, minimum and maximum intensity, standard deviation, skewness, and kurtosis. As one of textural features, histogram could provide a measure of intralesional heterogeneity $[5,14-15]$. Currently, there are many histogram studies on glioma [16-18], and DKI histogram analysis is becoming a hotspot [19-24]. The aim of this study was to compare the application of two DKI post-processing methods that DKE software and DKI histogram analysis in glioma grading, $I D H$ mutation typing, and evaluation of tumor heterogeneity.

\section{Methods}

\section{General information}

DKI was collected from September 2016 to September 2017. Before scanning, consent was obtained from the patients or their guardians. A total of 150 patients aged 14-70-years-old with single spaceoccupying lesions were recruited. Seventy-three patients had a surgical pathological diagnosis of glioma, including WHO II ( $n=21)$, WHO III $(n=13)$, and WHO IV ( $n=39)$, among whom 38 had IDH mutation status (IDH wildtype, $n=21$; IDH mutation, $n=17$ ).

\section{Equipment}

A Siemens Prisma 3.0 T (Prisma Siemens Healthcare, Erlangen, Germany) MRI scanner with a 64-channel head/neck coil was used. Conventional scans, enhanced scans, and DKI sequences that lasted a total of 30 minutes were performed on patients. The various sequence parameters are as follows: Axial and sagittal $T_{1}$-weighted imaging: $T R=250 \mathrm{~ms}, T E=2.46 \mathrm{~ms}$; Axial $\mathrm{T}_{2}$-weighted imaging: $\mathrm{TR}=4000 \mathrm{~ms}, \mathrm{TE}=$ $95 \mathrm{~ms}$; both sequences had similar FOV $=230 \mathrm{~mm} \times 230 \mathrm{~mm}$, number of slices $=20$, slice thickness $=5.0$ $\mathrm{mm}$, matrix $=256 \times 256$. A spin-echo echoplanar imaging sequence was used for the acquisition of DKI $(\mathrm{TR}=3500 \mathrm{~ms}, \mathrm{TE}=78 \mathrm{~ms} ; \mathrm{b}=0,500,1000,1500,2000,2500$; diffusion direction $=30$, slice thickness $=$ $5.0 \mathrm{~mm}$, number of slices $=20, \mathrm{FOV}=220 \mathrm{~mm} \times 220 \mathrm{~mm}$, matrix $=384 \times 384$, scan time $=9$ minutes and 1 second). Contrast-enhanced scan: a high-pressure injector was used for elbow vein injection of gadopentetate dimeglumine (Magnevist, Bayer Schering Pharma AG, Berlin, Germany) at a dose of 0.1 $\mathrm{mmol} / \mathrm{kg}$ bodyweight and an injection speed of $2.0 \mathrm{ml} / \mathrm{s}$. The $\mathrm{T}_{1}$ volume interpolated breath-hold sequence (TR $=630 \mathrm{~ms}$, TE $=9.3 \mathrm{~ms}$ ) was used for dynamic enhanced acquisition in the axial plane for six cycles. The sagittal plane 3D-T 1 MPRAGE (magnetization-prepared rapid gradient echo) sequence was used for the delayed enhanced scan, with TR $\ 2300 \mathrm{~ms}$, TE $=2.32 \mathrm{~ms}$, slice thickness $=0.9 \mathrm{~mm}$, number of slices $=179, \mathrm{FOV}=240 \mathrm{~mm} \times 240 \mathrm{~mm}$, matrix $=256 \times 256$, and a scan time of 5 minutes and 21 seconds, and axial and coronal post-contrast $\mathrm{T}_{1}$ MPRAGE images were reconstructed to include the whole brain with section thickness $=5 \mathrm{~mm}$ and intersection gap $=1 \mathrm{~mm}$. 


\section{Image Analysis}

The raw DKI images were imported into the DKE [9] software for preprocessing to obtain MK, RK, AK, mean diffusivity (MD), and fractional anisotropy (FA) graphs. Under the guidance of two experienced magnetic resonance physicians and by using the $\mathrm{T}_{2} \mathrm{WI}$ and $\mathrm{T}_{1}$ contrast-enhanced scan images as reference, we manually selected ROIs in continuous solid tumor regions in the MRIcron software by avoiding cystic changes, bleeding, necrosis, calcifications, and regions close to the blood vessels and cerebrospinal fluid. The entire solid tumor component of the glioma was selected as ROI (Figure 1) and the mean values of the corresponding parameters were calculated. At the same time, contralateral normal-appearing white matter (NAWM) from the same slice as the tumor ROI was selected and the parameters were compared to obtain corrected MK, RK, AK, FA, and MD values. Two radiologists with 15 years of experience in head and neck MRI (JB and GA), who were blinded to clinical information and histopathological results, delineated the ROls of all study subjects.

DWI obtained from the DKI scans were imported into the preset MATLAB platform for histogram analysis. The ROls selected by histogram analysis were consistent with those drawn in the MRIcron software (Figure 1) to obtain the minimum, maximum, mean, skewness coefficient, kurtosis coefficient, and $25^{\text {th }}$, $50^{\text {th }}, 75^{\text {th }}$, and $95^{\text {th }}$ percentiles for the Kapp and Dapp [25].

\section{Statistical Analysis}

Statistical Package for the Social Sciences, Version 21.0 (SPSS, IBM, Chicago, USA) was used for statistical analysis in this study, and $a=0.05$ was used as the test criterion. The MK, AK, RK, FA, and MD values for WHO Grade II, III, and IV gliomas, and the minimum, maximum, mean, skewness coefficient, kurtosis coefficient, and the $25^{\text {th }}, 50^{\text {th }}, 75^{\text {th }}$, and $95^{\text {th }}$ percentiles of the Kapp and Dapp values obtained from the MATLAB histogram analysis are expressed as mean \pm standard deviation ( $x \pm s$ ) for inter-group comparison of differences. When required, normalizing transformation (Bloom normalizing transformation) was carried out so that the data conformed to the requirements of the parametric tests. One-way ANOVA and the Least-Square Differential test were used for pairwise inter-group comparison. ROC curves were plotted, the AUC-ROC was calculated, and the threshold values for valid parameters were predicted.

\section{Results}

All the gliomas in this study located in the cerebrum, and the maximum tumor diameter ranged from $20 \mathrm{~mm}$ to $81 \mathrm{~mm}$. The results obtained from the two DKI post-processing methods are as follows.

Table 1 shows the distribution of various DKI parameters that were obtained using the DKE software for glioma grades. When DKI parameters were compared pairwise based on glioma grade (WHO II, III, and IV), there were significant differences in MK values among the different grades $(P<0.001)$ (Table 2). The 
differences in the AK value between WHO grades II and IV and between WHO III and IV were statistically significant $(P<0.05)$; however, the difference between WHO II and III was not significant $(P=0.832)$. There were no significant differences in RK, MD, and FA values in the pairwise comparison of various glioma grades $(P>0.05)$.

Table 1

The parameters of DKI calculated by DKE in glioma WHOQ-X

\begin{tabular}{llll}
\hline Parameter $(\overline{\mathrm{x}} \pm \mathrm{s})$ & WHO II & WHO III & WHO IV \\
\hline MK & $0.422 \pm 0.12$ & $0.724 \pm 0.08$ & $0.966 \pm 0.11$ \\
AK & $0.771 \pm 0.24$ & $0.774 \pm 0.24$ & $0.932 \pm 0.16$ \\
RK & $0.673 \pm 0.37$ & $0.699 \pm 0.099$ & $0.704 \pm 0.71$ \\
MD $\left(\times 10^{-3} \mathrm{~mm}^{2 / \mathrm{s})}\right.$ & $0.63 \pm 0.18$ & $0.58 \pm 0.1$ & $0.56 \pm 0.12$ \\
FA & $0.22 \pm 0.11$ & $0.17 \pm 0.09$ & $0.20 \pm 0.08$ \\
\hline
\end{tabular}

Table 2

The MK to differentiate glioma grades

\begin{tabular}{lccc}
\hline & WHO II and III & WHOIII and IV & WHO II and IV \\
\hline T value (t) & 8.05 & 7.17 & 17.71 \\
P value $^{*}$ & $<0.001$ & $<0.001$ & $<0.001$ \\
Cut-off value & 0.587 & 0.838 & 0.665 \\
Sensitivity & $100 \%$ & $92.3 \%$ & $100 \%$ \\
Specificity & $95.2 \%$ & $92.3 \%$ & $100 \%$ \\
AUC & 0.996 & 0.953 & 1.0 \\
\hline
\end{tabular}

${ }^{*}$ P value $<0.05$ as the inspection standard.

The minimum, maximum, mean, skewness, kurtosis, and 25th, 50th, 75th, and 95th percentiles of the Kapp and Dapp values were obtained through histogram analysis. When gliomas were separated into two 
groups, including high-grade (WHO III and IV) and low-grade (WHO II) glioma, the differences in the maximum, mean, standard deviation, 75th percentile (C75), and 95th percentile (C95) Kapp values and the minimum, mean, skewness, and the 25th percentile (C25), 50th percentile (C50), C75, and $\mathrm{C} 95$ of the Dapp values showed statistically significant differences $(P<0.05)$. When a pairwise comparison of WHO II, III, and IV gliomas was performed, the afore mentioned Kapp parameters showed statistically significant differences between WHO II and III, and WHO II and IV $(\mathrm{P}<0.05)$; however, there were no significant differences between WHO III and IV. The afore mentioned Dapp parameters showed significant differences between WHO II and IV $(P<0.01)$; however, the minimum and C25 Dapp values showed significant differences between WHO II and III $(P=0.049)$. There were no significant differences between WHO III and IV in the Kapp and Dapp histogram parameters. As seen in Tables 3 and 4 and the ROC curves (Fig. 2), the best Kapp histogram parameter was C75, and the best Dapp histogram parameter was the minimum Dapp.

Table 3

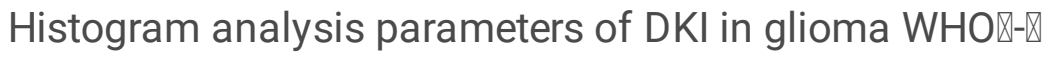

\begin{tabular}{llll}
\hline Parameter $(\overline{\mathrm{x}} \pm \mathrm{s})$ & WHO II & WHO III & WHO IV \\
\hline Kapp max & $4.37 \pm 0.2$ & $7.76 \pm 4.85$ & $10.65 \pm 2.78$ \\
Kapp mean & $0.42 \pm 0.19$ & $0.657 \pm 0.29$ & $0.71 \pm 0.37$ \\
Kapp SD & $0.825 \pm 0.33$ & $1.242 \pm 0.56$ & $1.389 \pm 1.3$ \\
Kapp C75 & $0.426 \pm 0.42$ & $0.798 \pm 0.43$ & $1.01 \pm 0.56$ \\
Kapp C95 & $2.282 \pm 0.85$ & $3.51 \pm 1.62$ & $3.41 \pm 1.57$ \\
Dapp min ${ }^{*}$ & $0.734 \pm 0.21$ & $0.579 \pm 0.2$ & $0.524 \pm 0.16$ \\
Dapp mean & $1.0 \pm 0.3$ & $0.859 \pm 0.24$ & $0.787 \pm 0.24$ \\
Dapp skwness & $0.196 \pm 0.884$ & $0.448 \pm 1.17$ & $0.914 \pm 0.935$ \\
Dapp C25 & $0.966 \pm 0.27$ & $0.791 \pm 0.237$ & $0.699 \pm 0.234$ \\
Dapp C50* & $1.047 \pm 0.3$ & $0.857 \pm 0.24$ & $0.771 \pm 0.26$ \\
Dapp C75 & $1.137 \pm 0.35$ & $0.923 \pm 0.244$ & $0.855 \pm 0.27$ \\
Dapp C95 & $1.256 \pm 0.356$ & $1.38 \pm 0.256$ & $1.027 \pm 0.3$ \\
\hline
\end{tabular}

* Dapp value unit $\left(\times 10^{-3} \mathrm{~mm}^{2} / \mathrm{s}\right)$. 
Table 4

The histogram ananlysis to differentiate glioma grades

\begin{tabular}{lccccccccccccc}
\hline & Kapp & Kapp & Kapp & Kapp & Kapp & Dapp & Dapp & Dapp & Dapp & Dapp & Dapp & Dapp \\
& max & meam & SD & C75 & C95 & min & mean & skewnes & C25 & C50 & C75 & C95 \\
& & & & & & & & s & & & & \\
\hline Cut-off value & 4.49 & 0.44 & 0.87 & 0.52 & 2.21 & $0.614^{*}$ & $0.889^{*}$ & -0.4 & $0.81^{*}$ & $0.87^{*}$ & $0.92^{*}$ & $1.06^{*}$ \\
Sensitivity & $77.6 \%$ & $85.7 \%$ & $79.6 \%$ & $87.8 \%$ & 83.7 & $80 \%$ & $80 \%$ & $80 \%$ & $80 \%$ & $80 \%$ & $80 \%$ & $80 \%$ \\
Specificity & $68 \%$ & $72 \%$ & $72 \%$ & $72 \%$ & $60 \%$ & $81.6 \%$ & $77.6 \%$ & $10.2 \%$ & $81.6 \%$ & $75 \%$ & $71.4 \%$ & $57.1 \%$ \\
AUC & 0.783 & 0.811 & 0.781 & 0.819 & 0.786 & 0.826 & 0.8 & 0.824 & 0.799 & 0.78 & 0.724 & 0.289 \\
\hline
\end{tabular}

※Dapp value unit $\left(\times 10^{-3} \mathrm{~mm}^{2} / \mathrm{s}\right)$.

Regarding IDH mutation status, 17 cases of IDH mutant glioma (WHO II $n=7$, WHO III $n=8$, WHO IV $n=2$ ) and 21 cases of wildtype IDH glioma (WHO II $n=3$, WHO III $n=1$, WHO IV $n=17$ ) could be accurately identified by MK $(0.65 \pm 0.22, P=0.004, A U C=0.79$, cut-off value $=0.71$, sensitivity $=81 \%$, specificity $=$ $76.5 \%), A K(0.73 \pm 0.25, P=0.020, A U C=0.67$, cut-off value $=0.75$, sensitivity $=81 \%$, specificity $=47.1 \%)$, Kapp mean $(0.45 \pm 0.13, P=0.006, A U C=0.80$, cut-off value $=0.49$, sensitivity $=81 \%$, specificity $=65 \%)$, C75 Kapp $(0.48 \pm 0.29, P<0.001, A U C=0.85$, cut-off value $=0.67$, sensitivity $=81 \%$, specificity $=70.6 \%)$, C95 Kapp (2.48 $\pm 0.71, P=0.017, A U C=0.73$, cut-off value $=2.39$, sensitivity $=76.2 \%$, specificity $=52.9 \%)$, but not by any Dapp, FA, or MD value.

\section{Discussion}

The diffusion of water molecules in human brain microstructure is affected by various cell membranes, proteins, and other factors [26-27]. Tumor tissue because of more nuclear atypia, higher cellular pleomorphism, more necrosis, and more microvascular proliferation [28] with a more complex diffusion status. As a non-Gaussianity diffusion modle DKI is thought to be present the microstructure of the tissue and close to the true state of tissue diffusion [29], and which parameters could be reflect the tissue diffusion quantitatively.

The results of our study demonstrated that $\mathrm{MK}$ could be used to differentiate WHO II, III, and IV gliomas, which is consistent with the results of other studies [8, 30-33]. Furthermore, the differences in AK values between WHO IV and II and between WHO IV and III were statistically significant, but those between WHO II and III were not significantly different. Consistent with the work of Zhao et al. [13], our results showed that MK and AK were also useful in identifying IDH mutation status. However, there were no significant differences in RK in our study between glioma grades or IDH mutation statuses, which is contrary to the results of other studies $[13,30]$. AK reflects the axonal integrity and density of fiber bundles and RK is assumed to reflect myelin integrity and axonal density [34]. However, MK is a measure of the overall 
kurtosis, which is computed as the average kurtosis along all uniformly distributed diffusion directions [34]. Gliomas grow and invade by destroying the axonal and fiber bundles, causing diffusion through complex tumor microstructures in a disorganized manner, which may cause more substantial changes in MK and AK than in RK.

In our study, the MD and FA values could not be used for glioma grading and identifying IDH mutation status. Currently, studies regarding MD and FA in grading and identifying IDH mutation status in glioma are controversial $[12-13,30,35]$. Therefore, the roles that MD and FA play in glioma require further investigation.

Although the Dapp and Kapp values obtained from the histogram analysis could not be used for the comparison between WHO III and IV gliomas, multiple Dapp and Kapp parameters showed statistically significant differences when comparing the high- and low-grade groups, consistent with the results of Hempel [19]. Furthermore, mean Kapp, C75 Kapp, and C95 Kapp could be used to predict IDH mutation status because the accuracy is higher than that of MK. Moreover, the Kapp standard deviation and Dapp skewness coefficient showed statistically significant differences between the high- and low-grade groups, demonstrating that these two markers have some use in determining the heterogeneity of consolidated tumors.

There are some limitations to our study. First, the sample size of our study was relatively small, especially the IDH gene data; the predictions of IDH mutation status could not exclude the influence of glioma grade. Second, several studies have demonstrated the utility of histogram analysis of the ADC in glioma grading, however, this parameter was not included in our analyses.

In conclusion, many of the DKI quantitative parameters obtained from the DKE software and histogram analysis could be used for the grading of gliomas (but identified the WHO III and IV grade glioma) and prediction of IDH mutation status. However, when come to glioma grading DKE software maybe batter than histogram, because its multiple parameters and higher diagnostic performance. Histogram is better at prediction of glioma IDH mutation status and display the heterogeneity.

\section{Abbreviations}

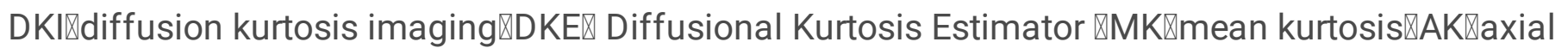

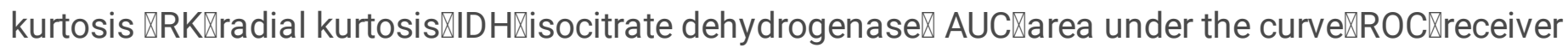

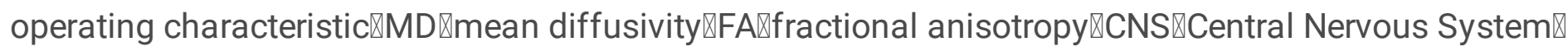

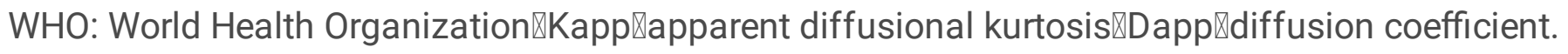

\section{Declarations}

\section{Ethics approval and consent to participate}


The Institutional Review Board of the First Affiliated Hospital of Zhengzhou University approved this retrospective study and waived the requirement for written informed consent due to its retrospective nature.

\section{Consent for publication}

Not applicable.

\section{Availability of data and materials}

Data related to the current study are available from the corresponding author on reasonable request.

\section{Competing interests}

The authors declare that they have no competing interests.

\section{Funding}

Medical tackling problems in science and technology plain program of Henan Province, China (201702070). Funding was utilized to collect, analysis and interpretation of data, and to write the manuscript. Funding body was not involved in design of the study.

\section{Authors' contributions}

JB: manuscript preparation, literature research, data acquisition, statistical analysis and manuscript editing; AKG: literature research, data analysis and statistical analysis; $\mathrm{YH}$ : data acquisition and data analysis; $\mathrm{YZ}$ and GHZ: data analysis; JL C and DX K: study conception and design, manuscript review and guarantor of integrity of the entire study. All authors read and approved the final manuscript.

\section{Acknowledgements}

Not applicable.

\section{References}

1. Louis DN, Perry A, Reifenberger G, et al. The 2016 World Health Organization Classification of Tumors of the Central Nervous System: a summary. Acta Neuropathol. 2016;131:803-20. 
2. Li F,Yue Zhang N, Wang, et al: Evaluation of the Prognosis of Neuroglioma Based on Dynamic Magnetic Resonance Enhancement. World neurosurgery,2020;S1878-8750.

3. Dahlrot RH. The prognostic value of clinical factors and cancer stem cell-related markers in gliomas. Dan Med J. 2014;61:B4944.

4. Ampie L, Choy W, Lamano JB, et al. Prognostic factors for recurrence and complications in the surgical management of primary chordoid gliomas: a systematic review of literature. Clin Neurol Neurosurg. 2015;138:129-36.

5. Chicklore S, Goh V, Siddique M, et al. Quantifying tumour heterogeneity in 18F-FDG PET/CT imaging by texture analysis. Eur J Nucl Med Mol Imaging. 2013;40:133-40.

6. Ganeshan B, Goh V, Mandeville HC, et al: Non-small cell lung cancer: histopathologic correlates for texture parameters at CT. Radiology,2013;266:326-336.

7. Jensen JH, Helpern JA, Ramani A, et al. Diffusional kurtosis imaging: The quantification of nongaussian water diffusion by means of magnetic resonance imaging. Magn Reson Med. 2005;53:1432.

8. Raab P, Hattingen E,Franz K, et al. Cerebral Gliomas: Diffusional Kurtosis Imaging Analysis of Microstructural Differences. Radiology. 2010;254:876-81.

9. Tabesh A, Jensen JH, Ardekani BA, et al. Estimation of tensors and tensor-derived measures in diffusional kurtosis imaging. Magn Reson Med. 2011;65:823-36.

10. Hui ES, Cheung MM, Qi L, et al. Towards better MR characterization of neural tissues using directional diffusion kurtosis analysis. Neurolmage. 2008;42:122-34.

11. Falk Delgado A, Nilsson $M$, van Westen $D$, et al. Glioma Grade Discrimination with MR Diffusion Kurtosis Imaging: A Meta-Analysis of Diagnostic Accuracy. Radiology. 2018;287:119-27.

12. Zhang J, Jiang J, Zhao L, et al. Survival prediction of high-grade glioma patients with diffusion kurtosis imaging. Am J Transl Res. 2019;11:3680-8.

13. Zhao J, Wang YL, Li XB, et al. Comparative analysis of the diffusion kurtosis imaging and diffusion tensor imaging in grading gliomas, predicting tumour cell proliferation and IDH-1 gene mutation status. J Neurooncol. 2019;141:195-203.

14. Just N. Improving tumour heterogeneity MRI assessment with histograms. $\mathrm{Br} \mathrm{J}$ Cancer. 2014;111:2205-13.

15. Raja R, Sinha N, Saini J, et al. Assessment of tissue heterogeneity using diffusion tensor and diffusion kurtosis imaging for grading gliomas. Neuroradiology. 2016;58:1217-31.

16. Jakab A, Molnár $\mathrm{P}$, Emri $\mathrm{M}$, et al. Glioma grade assessment by using histogram analysis of diffusion tensor imaging-derived maps. Neuroradiology. 2011;53:483-91.

17. Garzón B, Emblem KE, Mouridsen K, et al. Multiparametric analysis of magnetic resonance images for glioma grading and patient survival time prediction. Acta Radiol. 2011;52:1052-60.

18. Kim H, Choi SH, Kim JH, et al. Gliomas: application of cumulative histogram analysis of normalized cerebral blood volume on 3 T MRI to tumor grading. Plos One. 2013;8:e63462. 
19. Hempel JM, Schittenhelm J, Brendle C, et al. Histogram analysis of diffusion kurtosis imaging estimates for in vivo assessment of 2016 WHO glioma grades: A cross-sectional observational study. Eur J Radiol. 2017;95:202-11.

20. Qi XX, Shi DF, Ren SX, et al. Histogram analysis of diffusion kurtosis imaging derived maps may distinguish between low and high grade gliomas before surgery. Eur Radiol. 2018;28:1748-55.

21. Hempel JM, Brendle C, Bender B. Diffusion kurtosis imaging histogram parameter metrics predicting survival in integrated molecular subtypes of diffuse glioma: An observational cohort study. Eur $\mathrm{J}$ Radiol. 2019;112:144-52.

22. Xie H, Wu G: Application of Diffusion Kurtosis Imaging and Histogram Analysis for Assessing Preoperative Stages of Rectal Cancer. Gastroenterol Res Pract, 2018; 2018: 9786932.

23. Xu XQ, Ma G, Wang YJ, et al. Histogram analysis of diffusion kurtosis imaging of nasopharyngeal carcinoma: Correlation between quantitative parameters and clinical stage. Oncotarget. 2017;8:47230-8.

24. Li T, Hong Y, Kong D, et al. Histogram analysis of diffusion kurtosis imaging based on whole-volume images of breast lesions. J Magn Reson Imaging. 2020;51:627-34.

25. Rosenkrantz AB, Padhani AR, Chenevert TL, et al. Body Diffusion Kurtosis Imaging: Basic Principles, Applications, and Considerations for Clinical Practice. J Magn Reson Imaging. 2015;42:1190-202.

26. Rose S, Fay M, Thomas P, et al. Correlation of MRI-derived apparent diffusion coefficients in newly diagnosed gliomas with [18F]- fluoro-L-dopa PET: what are we really measuring with minimum ADC? AJNR. 2013;34:758-64.

27. Maier SE, Sun Y, Mulkern RV. Diffusion imaging of brain tumors. NMR Biomed. 2010;23:849-64.

28. Tietze A, Hansen MB, Ostergaard L, et al. Mean diffusional kurtosis in patients with glioma: initial results with a fast imaging method in a clinical setting. AJNR. 2015;36:1472-8.

29. Jensen JH, Helpern JA, Ramani A, et al. Diffusional kurtosis imaging: the quantification of nongaussian water diffusion by means of magnetic resonance imaging. Magn Reson Med. 2005;53:1432-40.

30. Van Cauter S, Veraart J, Sijbers J, et al. Gliomas: diffusion kurtosis MR imaging in grading. Radiology. 2012;263:492-501.

31. Lee HY, Na DG, Song IC, et al. Diffusion-tensor imaging for glioma grading at 3-T magnetic resonance imaging: analysis of fractional anisotropy and mean diffusivity. J Comput Assist Tomogr. 2008;32:298-303.

32. Bai Y, Lin Y, Tian J, et al. Grading of gliomas by Using Monoexponential, Biexponential, and stretched exponential Diffusion-weighted MR imaging and Diffusion Kurtosis MR imaging. Radiology. 2016;278:496-504.

33. Tietze A, Hansen MB, Østergaard L, et al. Mean Diffusional Kurtosis in Patients with Glioma: Initial Results with a Fast Imaging Method in a Clinical Setting. AJNR. 2015;36:1472-8. 
34. Wu EX, Cheung MM. MR diffusion kurtosis imaging for neural tissue characterization. NMR Biomed. 2010;23:836-48.

35. Jiang R, Jiang J, Zhao L, et al. Diffusion kurtosis imaging can effciently assess the glioma grade and cellular proliferation. Oncotarget. 2015;6:42380-93.

\section{Figures}
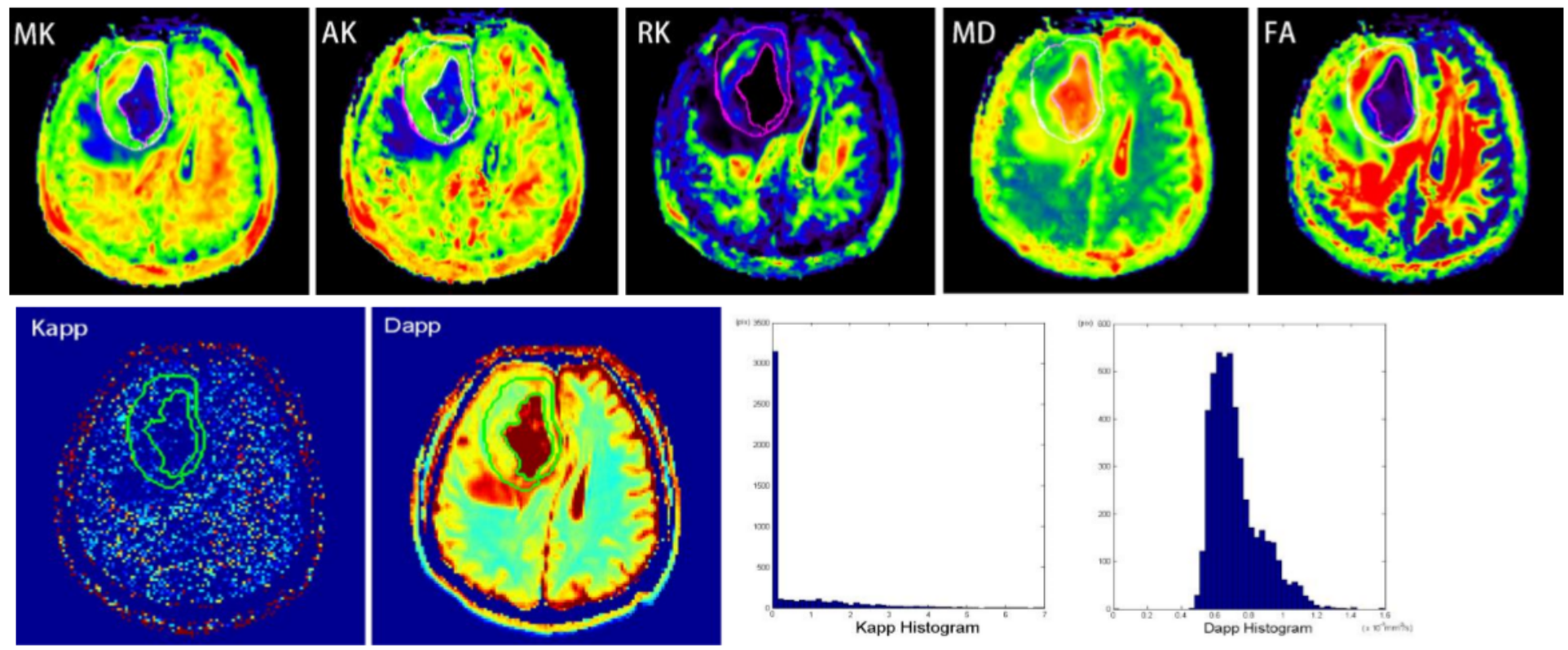

Figure 1

A 51-year old man with glioblastoma, IDH wild type, Whose DKI parameters and ROI site
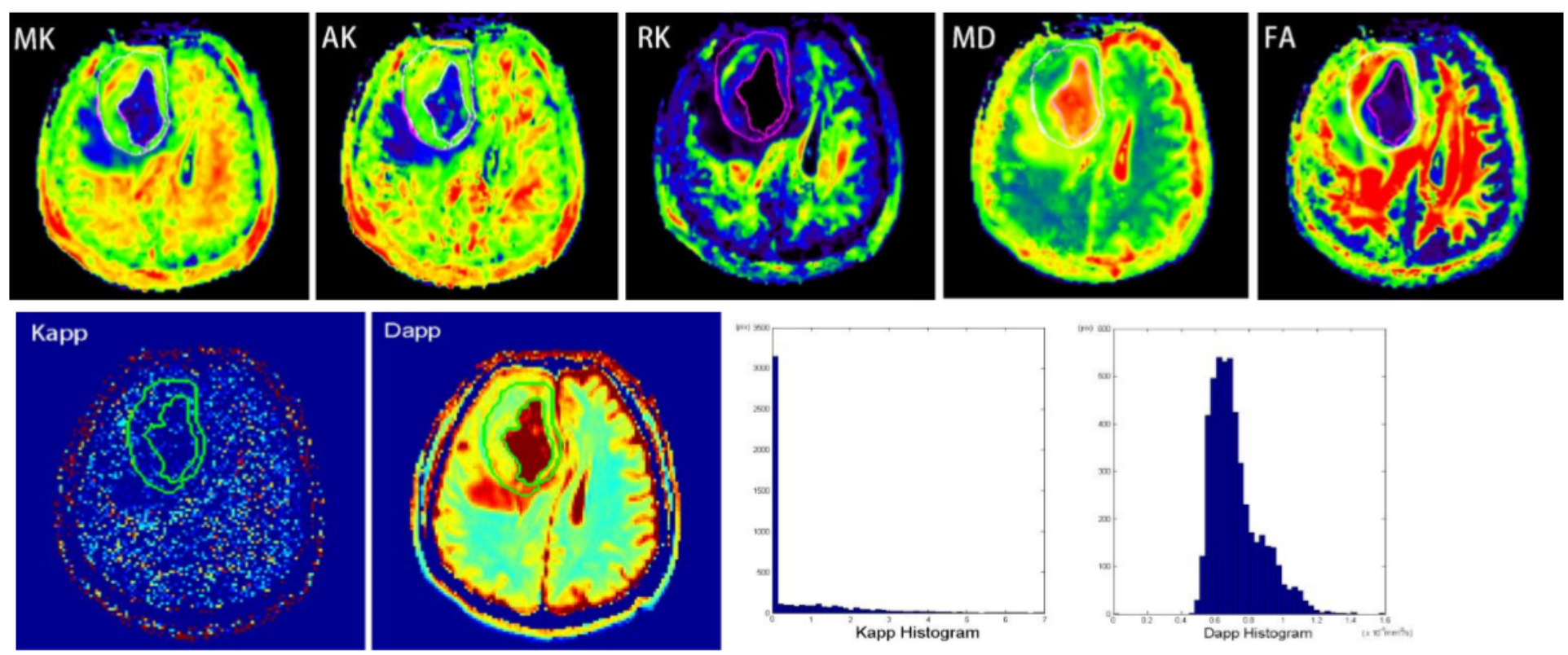

Figure 1 
A 51-year old man with glioblastoma, IDH wild type, Whose DKI parameters and ROI site
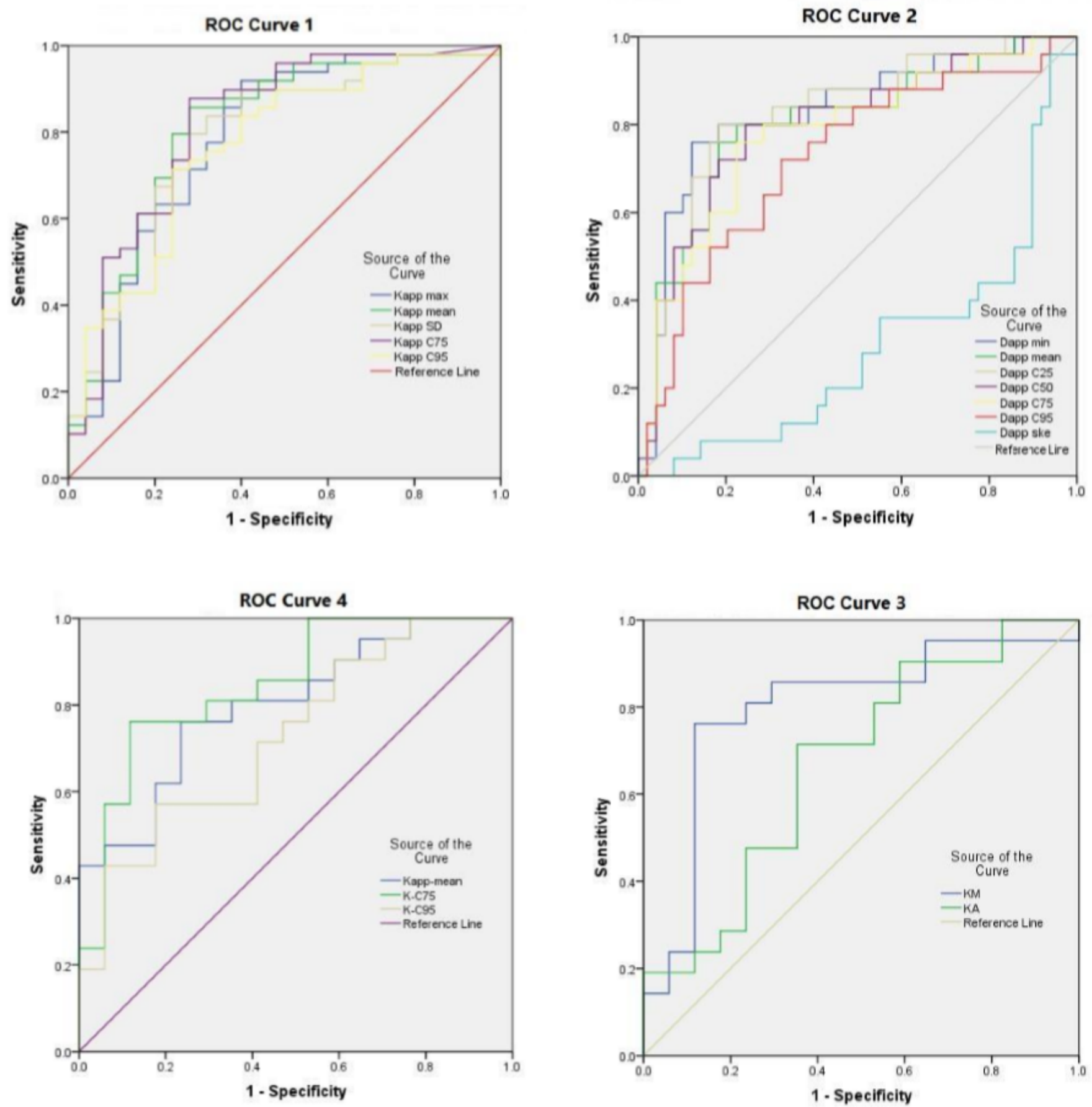

Figure 2

ROC Curve 1 is Kapp useful value for identifying high- and low-grade glioma. ROC Curve 2 is Dapp useful value for identifying high- and low-grade glioma. ROC Curve 3 is Kapp useful value for identifying IDH mutation type of glioma. ROC Curve 4 is MK and KA for identifying IDH mutation type of glioma. 

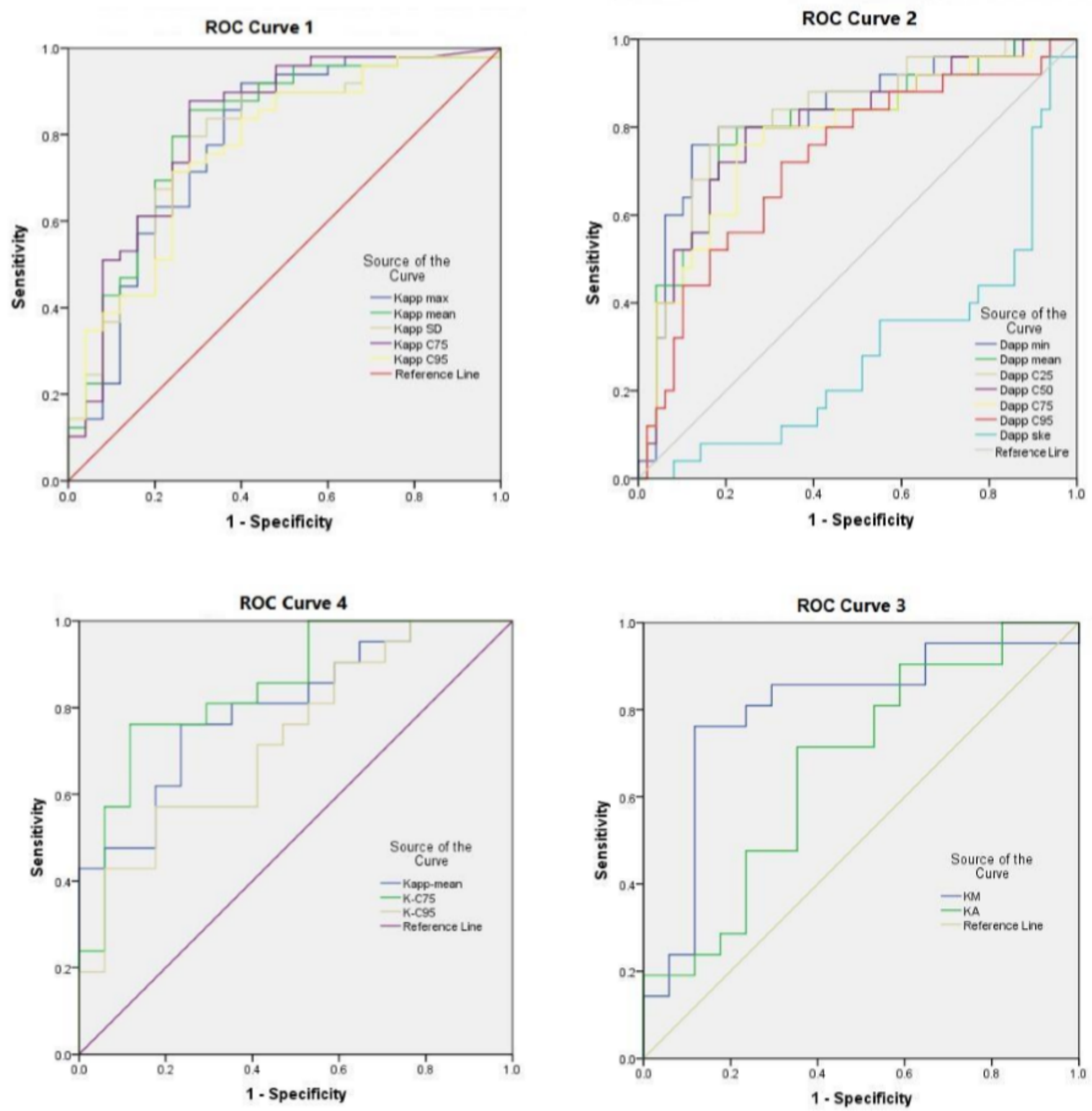

Figure 2

ROC Curve 1 is Kapp useful value for identifying high- and low-grade glioma. ROC Curve 2 is Dapp useful value for identifying high- and low-grade glioma. ROC Curve 3 is Kapp useful value for identifying IDH mutation type of glioma. ROC Curve 4 is MK and KA for identifying IDH mutation type of glioma. 